\title{
GENERAL DEGREE OF PERIODIC SPLINE FUNCTIONS
}

\author{
1. Manprit Kaur, \\ Deptt.of Mathematics, Gyan Ganga \\ College of Technology, Jabalpur
}

\author{
2. Arun Kumar, \\ Deptt. of Mathematics and Computer Science, \\ R.D.University, Jabalpur
}

\begin{abstract}
In this paper we consider spline function of general degree $\mathrm{m}$ which has the same area as the function does in each partition of the sub-intervals. The existence and uniqueness in general of spline functions also been studied and obtained the result.

$:$ In this paper we consider spline function of general degree, i.e.,of degree $m, \mathrm{~m}=2,3, \ldots$. We first give definitions and notations. We take $0=x_{0}<x_{1}<\ldots<x_{n-1}<x_{n}=1$ a subdivision of the interval $[0,1]$. The periodic spline function of degree $m$ was defined by Ahlberg, Nilson and Walsh [1] in the following way:

\section{DEFINITION 1 :}

A function $\varphi$ is said to be periodic spline function of degree $\mathrm{m}$ if it satisfies the following conditions:

( a ) In each sub-interval $\left[x_{i-1}, x_{i}\right], \mathrm{i}=1,2, \ldots, \mathrm{n}$, the function $\varphi$ coincides with a polynomial of degree at most $m$ i.e., $\varphi(x) \in \pi_{m}$; ( b ) its derivatives upto $\mathrm{m}-1$ order are continuous, i.e., $\varphi \in C^{(m-1)}[0,1]$;

( c ) $\varphi$ holds the boundary conditions; $\varphi^{(j)}(0)=\varphi^{(j)}(1), \mathrm{j}=$ $0,1, \ldots, \mathrm{m}-1$.

H. ter. Morsche [10] had defined the periodic spline function of degree $\mathrm{m}$ which is as follows:
\end{abstract}

\section{DEFINITION 2}

By $S(m, n)$ we denote the set of spline functions $\phi$, defined on $[0, \infty)$, that have the following properties :

(a) The restrictions of $\phi$ to an arbitrary sub-interval $\left[x_{i-1}, x_{i}\right]$ , i $=1,2, \ldots, \quad x_{i}=i h, \quad$ belongs to $\pi_{m}$;

(b) $\phi \in C^{m-1}[0, \infty)$.
The set of periodic spline functions of degree $\mathrm{m}$ corresponding to the uniform sub-division of the interval $[0,1]$ into n sub-intervals will be denoted by $S_{0}(m, n)$.

\section{DEFINITION 3:}

Truncated Power Function: The truncated power function is $x_{+}^{m}$. It is defined by

$$
x_{+}^{m}=
$$

[Sorry. Ignored lbegin $\{$ cases $\}$... lend $\{$ cases $\}$ ]

where $m$ is a positive real number.

\section{INTRODUCTION}

Ahlberg, Nilson and Walsh [1] had obtained the result for arbitrary periodic function $f$ with period $l$. This states that there exists a unique periodic spline function of odd degree that interpolates $\mathrm{f}$ at the nodes $\mathrm{x}_{i}$. Further added that it may not hold in general for even degree. Even degree case was considered by Subbotin [11]. He established that the result holds good if the nodes are equally spaced with arbitrary periodic function $\mathrm{f}$, if the interpolation points are taken to be the mid-points of the sub-intervals $\left[x_{i-1}, x_{i}\right]$. Meir and Sharma $[8,9]$ considered the above study for cubic case with a view to have more flexibility in the interpolating points for the class of function $C^{2}[0,1]$. Specifically, if the interpolation points are denoted by $y_{1}, y_{2}, \ldots, y_{n} \quad$ and if $y_{i}=x_{i}-\lambda h \quad$ with $0 \leq \lambda \leq \frac{1}{3} \quad$ or $\frac{2}{3} \leq \lambda \leq 1$, then they established the existence and uniqueness of a periodic cubic spline function interpolating arbitrary element of $C^{2}[0,1]$ at the points $y_{i}$. About there convergence they proved the following theorem [9] : 
THEOREM A: Let $f(x) \in C^{2}[0,1]$ be 1-periodic, and let $\varphi(x) \in C^{2}[0,1]$ be the 1-periodic cubic spline with joints $x_{i}=i / n$, satisfying $\varphi\left(\frac{i+\lambda-1}{n}\right)=f\left(\frac{i+\lambda-1}{n}\right)$, $i=1,2, \ldots, n$, where $\quad 0 \leq \lambda \leq \frac{1}{3} \quad$ or $\quad \frac{2}{3} \leq \lambda \leq 1 . \quad$ Then $\max _{x}|\varphi(x)-f(x)| \leq 15 \omega_{2}\left(\frac{1}{n}\right)$, where $\omega_{2}$ is the second modulus of continuity of $f$. Instead of considering point interpolation, Dikshit [5] studied certain area-matching of the spline function and the function in each sub-interval of the partition. Essentially he obtained the following [1978 ]

\section{THEOREM B:}

Let $f \in C^{2}[0,1]$ be a 1-periodic locally integrable function with respect to a non-negative measure $\mathrm{d} \mu$ satisfying $\mu(x+h)-\mu(x)=K \quad$ (constant).Suppose further that either $\int_{0}^{h} \alpha(x) d \mu>0 \quad$ or $\quad \int_{0}^{h} \alpha(h-x) d \mu>0$,where $\alpha(x)=3 x^{3}-6 h x^{2}+h^{3}$. Then there exists a unique $s(x) \in S(3, \Delta)$ satisfying the following conditions: $x_{i}$ $\int\{f(x)-s(x)\} d \mu=0$ $i=1,2, \ldots, n$, and

$x_{i-1}$

$s^{(r)}(0)=s^{(r)}(1)$,

$r=0,1,2 . \quad$ It was also shown that the result of Meir and Sharma [8,9] follows from the above theorem as particular case. Since this result does not cover the interpolation at the mid-points of the mesh so a separate result was proved by Dikshit ([5], Th.2, per Remark 2 ) which allows to consider only mid-points of a mesh as interpolatory condition. For every odd n, above result continues to hold if we assume the conditions

$$
\int_{0}^{h}\left(-4 x^{3}+6 h^{2} x-h^{3}\right) d \mu=0
$$

and $\int_{0}^{h} d \mu>0$ in place of

$$
\int_{0}^{h} \alpha(x) d \mu>0 \text { or }
$$

$\int_{0}^{h} \alpha(h-x) d \mu>0$, where $\alpha(x)=3 x^{3}-6 h x^{2}+h^{3}$.

A similar

result for area-matching was studied by Kumar and Govil [7]. They obtained the following

\section{:THEOREM C}

Let $\mathrm{f} \in \mathrm{L}[0,1]$. Then there exists a unique spline $\mathrm{s}(\mathrm{x}) \in S(3, \Delta)$ which bounds the same area as the function does, precisely,

$$
\int_{x_{i-1}}^{x_{i}} f(x) d x=\int_{x_{i-1}}^{x_{i}} s(x) d x, \quad \mathrm{i}=1,2, \ldots, \mathrm{n}, \text { if } s^{\prime \prime}(0)=s(1)=0
$$

and $\quad h(s(1-h)-s(h)=24 S(0)$.

The study of area-matching spline function was further investigated by Das ( Thesis 2004[6], Th.3.1( a ), ( b )) and obtained the following result :

\section{THEOREM D :}

( a ) Let $f \in G_{p}$ ( by $f \in G_{p}$ it is meant that $\mathrm{f}$ is integrable with respect to measure $d g$ and also it is periodic with period-1), then there exists a unique 1-periodic spline function $s \in S(3, \Delta)$ such that, for $0 \leq \alpha \leq 1$,

$$
\begin{aligned}
& \int^{x_{i}} s(x) d g+\alpha \int^{x_{i+1}} s(x) d g=\int_{x_{i}}^{x_{i}} f(x) d g+\alpha \int^{x_{i+1}} f(x) d g \\
& \begin{array}{llll}
x_{i-1} & x_{i} & x_{i-1} & x_{i}
\end{array} \\
& =1,2, \ldots, \mathrm{n} \text {,if } \\
& \int_{0}^{h} \mu(x) d g=\int_{0}^{h} \mu(h-x) d g=\delta_{a}>0,
\end{aligned}
$$

where $\mu(x)=3 x^{3}-6 h x^{2}+h^{3}$, and $\mathrm{n}$ is odd when $\alpha=1$. For $\alpha=1$ the results fails to exist if $\mathrm{n}$ is even. ( $\mathrm{b}$ ) Let $\beta, 0 \leq \beta \leq 4$ be zero of $\quad \int_{0}^{h} \sigma(\beta, x) d g=0$,

where

$\sigma(\beta, x)=$

$-\beta^{3}(h-x)^{3}+\beta^{2}\left(3 x^{3}-6 h x^{2}+4 h^{3}\right)-\beta\left(-3 x^{3}+3 h x^{2}+3 h^{2} x+h^{3}\right)+x^{3}$.

The above theorem is true, in case $\beta \neq 1$, if $\int^{h}\left\{-\beta^{2}(h-x)^{3}+\beta\left(4 x^{3}-9 h x^{2}+3 h^{2} x+3 h^{3}\right)-x^{3}\right\} d g=\delta_{b}>0, \quad$ and, 0

in case if $\beta=1$, iff $\mathrm{n}$ is odd. The results of Meir and Sharma [9] have been generalized by H.ter Morsche [10] for interpolating periodic splines for general degree. This result is contained in the following theorems :

\section{THEOREM E :}

Let $\mathrm{f}$ be a periodic function with period 1 . If the linear system

$$
\sum_{r=0}^{m}(m !)^{-1} M_{i-1+r} \sum_{j=0}^{r}(-1)^{j} m+2 j(r-j+\lambda)^{m}=h^{1-m} \Delta^{m-1} f\left(x_{i}\right),
$$

$i=1,2, \ldots, n, \quad$ in the unknowns $M_{0}, M_{1}, \ldots, M_{n-1}$, where $M_{n+k}=M_{k}$ for all $\mathrm{k}$, has a unique solution $M=\left(M_{0}, M_{1}, \ldots, M_{n-1}\right), \quad$ then there exists one and only one function $\phi \in S_{0}(m, n) \quad$ with $\phi\left(x_{i}\right)=f\left(x_{i}\right)(i=1,2, \ldots, n)$.

\section{THEOREM $F$}

: Let there be given a uniform subdivision $0=x_{0}<x_{1}<\ldots<x_{n-1}<x_{n}=1$ of the interval $[0,1]$ together with a periodic function $\mathrm{f}$ with period 1 . Furthermore, let the interpolation points $x_{i}$ be defined as, $x_{i}=y_{i}-\lambda h, \quad 0<\lambda<1$. Then there exists a uniquely determined periodic spline function $\phi \in S_{0}(m, n) \quad$ with the interpolation properties

$$
\phi\left(x_{i}\right)=f\left(x_{i}\right), \quad i=1,2, \ldots, n,
$$

in each of the following cases:

(i ) $\mathrm{n}$ is odd.

(ii ) $m$ is odd and $\lambda \neq \frac{1}{2}$.

(iii ) $\mathrm{m}$ is even and $\lambda \neq 0$ and $\lambda \neq 1$. 
The proof of the results obtained in Morsche[10] make use of the following interesting results:

\section{THEOREM G :}

Let,

$P_{m}(z, \lambda)=\sum_{r=0}^{m} a^{r} z^{r}$

(a) For all $\mathrm{z}$ and $\lambda$ one has

$$
P_{m}(z, \lambda)-z P_{m}(z, \lambda+1)=(m !)^{-1}(1-z)^{m+1} \lambda^{m} .
$$

(b) For all $\lambda$ and $z \neq 0$ one has

(c)

$$
P_{m}(z, \lambda)=z^{m} P_{m}\left(z^{-1}, 1-\lambda\right) .
$$

$\sum_{r=0}^{n-1} a_{r}=1$

$a_{r}=$

[Sorry. Ignored Vbegin $\{$ cases $\}$... lend $\{$ cases $\}]$

( d ) For $\mathrm{k}=0,1, \ldots, \mathrm{n}-1$ one has $a_{k} \geq 0$. ( e ) With respect to $\lambda, P_{m}(-1, \lambda) \quad$ is a polynomial of degree $m$ and there holds $P_{m}(-1, \lambda)=(m !)^{-1} 2^{m} E_{m}(\lambda), \quad$ where $E_{m}$ is the so called Euler polynomial of degree $\mathrm{m}$.( $\mathrm{f}$ ) For all $\lambda$ one has $\frac{d}{d \lambda} P_{m}(-1, \lambda)=2 P_{m-1}(-1, \lambda) . \quad(\mathrm{g})$ For $0<\lambda<1$ the polynomial $P_{m}(z, \lambda)$ has $m$ negative and distinct zeros. For $\lambda=0$ the polynomial $P_{m}(z, \lambda)$ has $\mathrm{m}-1$ negative and distinct zeros, while in addition $z=0$ is a zero.( $\mathrm{h}$ ) For even $\mathrm{m}$ the only zeros of $P_{m}(-1, \lambda) \quad$ on $[0,1]$ are $\lambda=0$ and $\lambda=1$. For odd $m$ the only zero of $P_{m}(-1, \lambda) \quad$ on $[0,1]$ is $\lambda=\frac{1}{2}$. The main object of this paper is to consider spline of general degree i.e., of degree $\mathrm{m}$ which has the same area as the function does in each partition of the sub-intervals. We prove the following:THEOREM : Let there be given a uniform subdivision $0=x_{0}<x_{1}<\ldots<x_{n-1}<x_{n}=1$ of the interval [0,1] together with a periodic function $f$ with period 1 . Then there exists uniquely determined periodic spline function $\phi \in S_{0}(m, n)$ for odd values of $\mathrm{n}$ satisfying $\int_{x_{i-1}}^{x_{i}} \phi(x) d x=\int_{x_{i-1}}^{x_{i}} f(x) d x$,

$i=1,2, \ldots, n$. We need the

following lemma, for proof of the theorem:LEMMA 1 : Let there be given an infinite sequence of real numbers $M_{0}, M_{1}, \ldots$. A function $\phi \in S(m, n)$ has the properties: $\quad \phi^{(m-1)}\left(x_{i}\right)=M_{i}$ $(\mathrm{i}=0,1,2, \ldots)$ if and only if $\phi$ can be written in the form $\phi(x)=p(x)+(m !)^{-1} \sum_{k=0}^{\infty} M_{k} \Delta^{2}\left(x-x_{k+1}\right)_{+}^{m}$,

where $p(x) \in \pi_{m-2}$ is an arbitrary polynomial.

The above lemma is contained in (cf [10], p.199).
LEMMA 2 : We have

$$
\sum_{j=0}^{m+2}(-1)^{m+2-j} m+2 j e^{(j-1) t}\left(\frac{d}{d t}\right)^{m+1}\left(\frac{e^{(m+3-j) t}}{1+e^{t}}\right)=0
$$

The lemma follows from (cf.[10],pg. 205) on taking $m=m+1$. The coefficient matrix of the equation (3.8) is

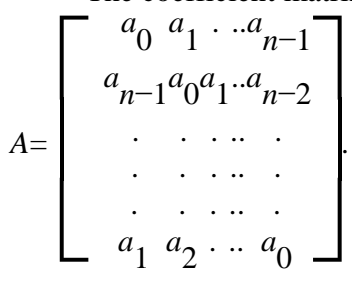
singular.

The solutions of the equations are unique if $A$ is not Let $Q$ be the circulant matrix, precisely $Q=C(0,1, \ldots, 0)$. We can write

(3.9) $A=a_{0} Q^{0}+a_{1} Q^{1}+\ldots+a_{n-1} Q^{n-1}$.

In order to obtain eigen values of the matrix of A we consider the polynomial $R_{m}(s)$. We have Morsche ([10], cf. p.204),

$R_{m}(s)(m+1) !=\sum_{j=0}^{m+2} \sum_{j=0}^{r}(-1)^{m-j}{ }_{m+2 j(j-r-1)^{m+1}} s^{r}$.

On changing the order of summation, we obtain

$$
\text { (3.10) } R_{m}(s)(m+1) !=\sum_{j=0}^{m+2}(-1)^{m-j}{ }_{m+2 j} \sum_{r=j}^{m+2}(j-r-1)^{m+1} . s^{r},
$$

We substitute

$$
r-j=l \text { and } s=-e^{t} \text {. }
$$

The above equation becomes

$$
\begin{gathered}
R_{m}(s)(m+1) !=-\sum_{j=0}^{m+2}(-1)^{m-j}{ }_{m+2 j} \sum_{l=0}^{m+2-j}(l+1)^{m+1} \cdot\left(-e^{t}\right)^{l+j} \\
=-\sum_{j=0}^{m+2}(-1)^{j} m+2 j \sum_{l=0}^{m+2-j}(l+1)^{m+1} \cdot\left(-e^{t}\right)^{l+j} \\
=-\sum_{j=0}^{m+2}(-1)^{j}\left(m+2 j(-1)^{j} e^{(j-1) t} \sum_{l=0}^{m+2-j}(l+1)^{m+1}(-1) e^{(l+1) t}\right. \\
=-\sum_{j=0}^{m+2} m+2 j e^{(j-1) t} \sum_{l=0}^{m+2-j}(-1)^{l}(l+1)^{m+1} e^{(l+1) t} \\
=-\sum_{j=0}^{m+2} m+2 j e^{(j-1) t}\left(\frac{d}{d t}\right)^{m+1} \sum_{l=0}^{m+2-j}(-1)^{l} e^{(l+1) t}
\end{gathered}
$$




\section{Error!}

$=-\sum_{j=0}^{m+2} m+2 j e^{(j-1) t}\left(\frac{d}{d t}\right)^{m+1}\left(\frac{e^{t}}{1+e^{t}}\right)$,

by Lemma 2 .

Therefore

$$
R_{m}(s)=\frac{\left(1+e^{t}\right)^{m+2}}{(m+1) !} e^{-t}\left(\frac{d}{d t}\right)^{m+1}\left(\frac{e^{t}}{1+e^{t}}\right) .
$$

Now we see zeros of $R_{m}(s)$. It is direct to see that the zeros of $R_{m}(s)$ are zeros of

$$
e^{-t}\left(\frac{d}{d t}\right)^{m+1}\left(\frac{e^{t}}{1+e^{t}}\right)=K_{m+1}(t),
$$

say. We have

$$
K_{1}(t)=\frac{1}{\left(1+e^{t}\right)^{2}}
$$

and it has no zero. Further,

$$
K_{2}(t)=-\frac{-1+e^{t}}{\left(1+e^{t}\right)^{3}},
$$

and this is zero only for $t=0$ i.e., $s=-1$. Next

$$
K_{3}(t)=-\frac{-1+4 e^{t}-e^{2 t}}{\left(1+e^{t}\right)^{4}},
$$

it has got only two real zeros, namely, $e^{t}=2+\sqrt{3}, 2-\sqrt{3}$ i.e., $s=-(2+\sqrt{3}),-(2-\sqrt{3})$.

Now we show by induction that $K_{m+1}(t)$ has got $m$ real zeros. We have

$$
K_{l+2}(t)=K_{l+1}^{\prime}(t)=\frac{d}{d t} K_{l+1}(t) .
$$

Suppose, $K_{l+1}(t)$ has got $l$ zeros. By Rolle's theorem between any two real zeros there exists one real zero. Hence, derivative of $K_{l+2}(t)$ has got $l-1$ real zeros between the zeros of $K_{l+1}(t)$.

Further , $K_{l+1}(t)$ becomes zero for $t \rightarrow \pm \infty$. Hence $K_{l+2}(t)$ has got one zero left to the zero of $K_{l+1}(t)$ and one zero between the right zero of $K_{l+1}(t)$ and 0 . Thus $K_{l+2}(t)$ has got $l+1$ negative zeros.
Hence $K_{m+1}(t)$ has got $m$ real zeros.

Now we proceed to locate eigen values of A. It is known that the $n$ eigen values of $Q$ are the roots of unity i.e., $\omega_{k}=e^{\frac{2 \pi i k}{n}}, \mathrm{k}=0,1, \ldots, \mathrm{n}-1$. From above equation it follows that the eigen values of $\mathrm{A}$ correspond to $R(s)$ for $s=\omega_{k}$, $\mathrm{k}=0,1, \ldots, \mathrm{n}-1$. One of the root $\omega_{k}$ becomes real i.e., only for even $n$. Thus $\mathrm{A}$ has possibly an eigen value zero for even $n$. For odd $n$ there is no eigen value zero. Hence, $\mathrm{A}$ is invertible for odd $n$. This proves the theorem.

\section{REFERENCES:}

[1] Ahlberg, J. H. , Nilson , E. N. and Walsh , J. L., The theory of splines and their applications, Academic Press, (New York, 1967).

[2] de Boor, C., Best approximations properties of splines of odd-degree, J. Math. Mech. 12,(1968),747-749.

[3] Das, V.B., Spline interpolation by lower degree polynomials using area matching condition, $\mathrm{Ph} . \mathrm{D}$. Thesis, Rani Durgawati Uni. (2004), Jabalpur.

[4] Demko, S., Interpolation by Quadratic Splines, J. Approx. Theory, 23(1978), 392-400.

[5] Dikshit, H.P., On cubic interpolatory Splines, J. Approx. Theory, 22(1978), 105-107.

[6] Kumar and Das, Convergence of Cubic Piecewise Function, Journal of Computat1ional and Applied Mathematics , 194(2006),388-394.

[7] Kumar and Govil, L.K., On Deficient Cubic Splines Interpolants, J. Approx. Theory, 68(1992), 175-182.

[8] Meir, A. and Sharma, A., A Degree of Approximation, J. Math. Mech., 15(1966), 759-768.

[9] Meir, A. and Sharma, A., Convergence of a class of interpolatory splines, J. Approx. Theory, 1(1968), 243-250.

[10] Morsche H.ter., On the existence and convergence of interpolating periodic spline functions of arbitrary degree, Deptt. of mathematics, Tech.Uni. Eindhoven, The Netherlands, 197-214.

[11] Subbotin, Yu. N., Piecewise Polynomial (spline) Interpolation, Math. Notes, 1(1967), 41-45 